\title{
Ultrasonic assessment of gastric emptying in the preterm infant
}

\author{
S J Newell, S Chapman, I W Booth
}

\begin{abstract}
The study of gastric emptying in the preterm infant has been hampered by the absence of a suitable, valid technique. We have evaluated gastric antral clearance using serial ultrasonic measurement of the antral cross sectional area (ACSA). The study was easy to perform, providing successful results in 15 of 17 low birthweight infants, with feed volumes of 8-31 $\mathrm{ml} / \mathrm{kg}$. ACSA was closely related to intragastric volume (mean (range) $r=0.96$ $(0 \cdot 89-0 \cdot 99))$. Measurements were reproducible: mean (SE) coefficient of variation was $7 \cdot 7 \%(1 \cdot 1)$. Gastric antral clearance was observed as a fall in ACSA that began shortly after completion of the feed and returned to prefeed values at a variable rate. The pattern of gastric antral transit was entirely consistent with recognised patterns of gastric emptying and half gastric antral clearance times ranged from 20-63 minutes. Ultrasonic measurement of gastric antral clearance is a new technique allowing the study of gastric emptying in the preterm infant.

(Arch Dis Child 1993; 69: 32-36)
\end{abstract}

Successful enteral nutrition of the very low birthweight infant is both an important objective and a considerable challenge, for while digestive and absorptive capacity may be sufficient to handle full milk feeds, impaired gastrointestinal motility often limits the introduction of milk. Clinically the infant is said not to 'tolerate' feeds and is reported to have large gastric residual volumes, vomiting, or abdominal distension. Efficient gastric emptying is clearly a major controlling influence, yet few data exist from studies of gastric emptying in very low birthweight infants. This is largely due to the absence of a method of assessing gastric emptying suitable for use in the compromised neonate within an intensive care setting.

Gastric emptying may be measured in children and adults using radiographic contrast studies, scintigraphy, and dye dilution techniques that are not suitable for the infant on the neonatal intensive care unit. ${ }^{1}$ We have previously reported the successful use of transabdominal impedance for the measurement of gastric emptying, ${ }^{2}$ but because of movement artefact the method did not translate well for use in the low birthweight infant. Recent reports have focused on the use of ultrasound to measure gastric emptying. In initial studies total gastric volume was estimated using multiple ultrasound images along the length of the stomach. ${ }^{34}$ A greatly simplified technique using serial measurement of antral cross sectional area (ACSA) ${ }^{5}$ has since been used in children ${ }^{6} 7$ and validated against scintigraphic measurement of gastric emptying in adults. ${ }^{8}$ We have modified the technique using serial measurements of ACSA to determine gastric antral clearance. In this study we aimed to examine the applicability, reproducibility, and validity of our technique for the assessment of gastric emptying in the low birthweight infant.

\section{Subjects and methods}

The study was carried out on the regional neonatal unit at the Birmingham Maternity Hospital. Low birthweight infants were selected if they satisfied the following criteria: preterm delivery, absence or complete recovery from respiratory illness, no evidence of gastrointestinal disease, no drug treatment, and were receiving full feeds of breast milk or infant formula at a minimum of $150 \mathrm{ml} / \mathrm{kg} /$ day.

Seventeen consecutively available infants whose parents gave consent for study were recruited. Median (range) birth weight was 1800 (680-2300) g, gestation 32 (25-36) weeks, and postnatal age 2 (1-8) weeks. Median (range) feed volume was 180 $\mathrm{ml} / \mathrm{kg} / \mathrm{day}(150-200)$ given as two, three, or four hourly bolus by nasogastric tube with a median feed volume of $26 \mathrm{ml} / \mathrm{kg}$ (8-31). Five infants were receiving their mother's expressed breast milk, and 12 a standard whey dominant infant formula (Cow and Gate Premium). The protocol was approved by the district ethical committee.

\section{ANATOMY OF THE ACSA}

The gastric antrum varies in circumference along its length. It is therefore imperative that the ACSA is measured using constant anatomical reference points (fig 1). The transducer was held perpendicular to the anterior abdominal wall, and a longitudinal section of the gastric antrum was obtained along the length of the abdominal aorta at the level of the superior mesenteric artery. This provided a cross section of the gastric antrum distal to the incisura.

\section{ULTRASOUND IMAGING}

All studies were performed using real time two dimensional ultrasound. The Hewlett Packard ultrasound imaging system (model 77020A) was used with a $5 \mathrm{MHz}$ sector scanning probe (model 21210A). Software available with the 


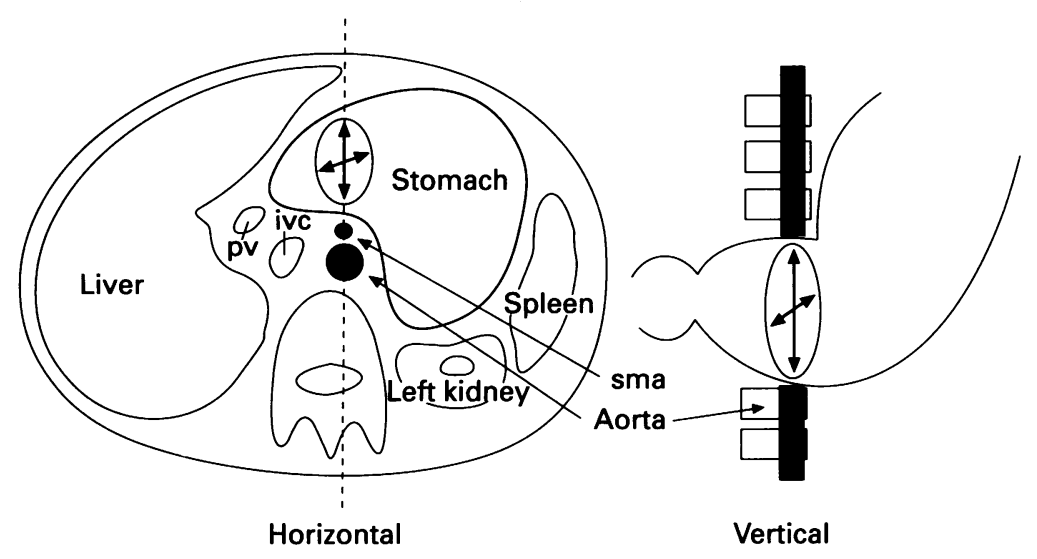

Figure 1 Anatomy of ACSA between the midline anteriorly and the aorta at the level of the aorta and superior mesenteric artery (sma); ivc: inferior vena cava, pv: portal vein.

machine allowed the image to be frozen on the screen, the circumference of the gastric antrum marked with a steerable cursor, and the calculation of the ACSA.

\section{STUDY TECHNIQUE}

Infants were studied at times when feeds were due routinely. Other nursing care was completed before the study. The stomach was then emptied by aspirating the nasogastric tube and the infant placed in the right lateral position for the remainder of the study. This ensured that any residual air in the stomach was displaced to the fundus where it would not interfere with imaging of the antrum. Clothing over the upper abdomen was loosened in order to minimise disturbance to the infant during the study.

Serial measurements of ACSA were made before, during, and after administration of the feed. Before the infant's next feed the nasogastric tube was again aspirated to determine any residual volume. The study comprised three parts:

\section{(i) Relationship between intragastric volume and} ACSA

To test the hypothesis that the ACSA is proportional to intragastric volume, in half the subjects $(n=8)$ the feed volume was administered stepwise over a period of $2-4.5$ minutes and measurements were made before the feed and after administration of $25,50,75$, and $100 \%$ of the feed volume. The correlation between gastric volume and ACSA was calculated. In the remaining infants the feed was given as a bolus over one minute.

(ii) Reproducibility of the measurement of ACSA All ultrasound images were recorded on videotape for later assessment. In the first 10 infants studied, 10 measurements were made of ACSA over a period of 50-90 seconds during the early phase of gastric emptying. The measurements were all made by one observer (SJN) who was blind to patient identity. In order to prevent recall of the previous measurements in any baby, the first of the 10 measurements was made on patient 1 then on patient 2 and so on to patient 10 , then returning to patient 1 for her second measurement. The coefficient of variation was calculated (SD/mean $\times 100 \%$ ) for each patient and for the group. Confidence interval analysis allowed calculations of the $95 \%$ intervals for any single results.

(iii) Pattern of gastric antral clearance during gastric emptying

At the end of each feed a measurement of ACSA allowed calculation of the change in area during the feed. Serial measurements were then made on each infant at approximately 10 minute intervals once the feed was completed. This allowed both observation of the pattern of gastric antral clearance, and calculation of $50 \%$ delta ACSA: the time taken for the ACSA to fall by half of the maximal change seen during gastric filling.

\section{Results}

The technique was simple to perform. Satisfactory recordings were obtained in 15 of 17 subjects. In two, the gastric antrum could not be imaged because of gas bubbles in the stomach. One of these infants had been crying before the study and the other appeared to have swallowed air during the tube feed. In no infant was vomiting, distress, or other adverse reaction noted during the study.

\section{(i) Relationship between intragastric volume and} ACSA

The median (range) gastric ACSA before introduction of the feed was $0.18 \mathrm{~cm}^{2}$ $(0 \cdot 11-0 \cdot 89)$. The initial ACSA was not related to the baby's size. The relationship between intragastric milk volume and the increase in ACSA was examined after $25,50,75$, and $100 \%$ of the feed had been given. A strong linear relationship was seen between gastric volume and ACSA (fig 2). Correlation coefficients for individual infants were very high

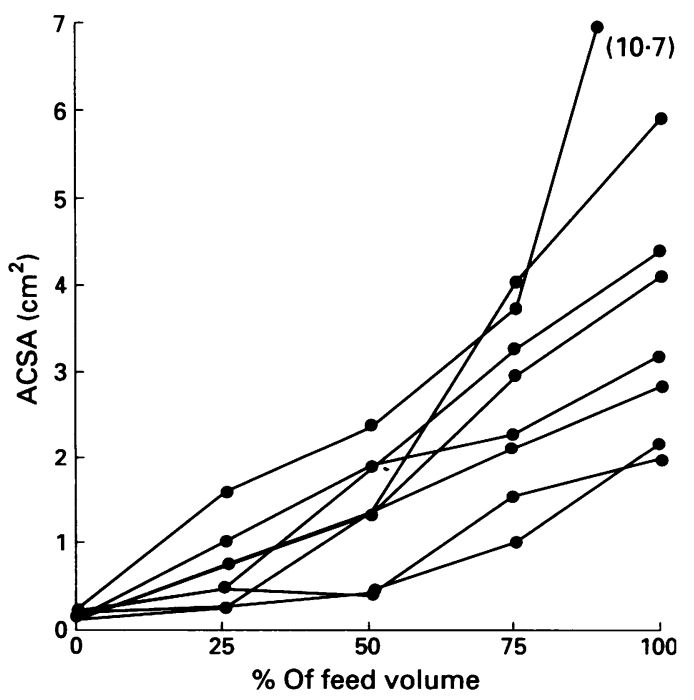

Figure 2 Relationship between ACSA $\left(\mathrm{cm}^{2}\right)$ and gastric volume during introduction of the feed. Mean (range) correlation coefficient, $r=0.96(0.88-0.99) ; p<0.001$. 


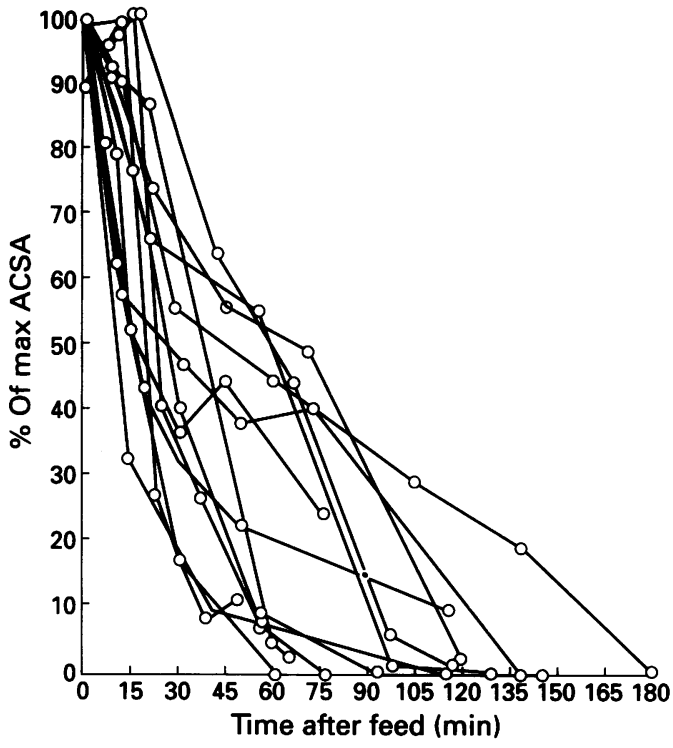

Figure 3 Gastric antral transit: fall in ACSA during gastric emptying. Median half gastric antral transit time (range): $28(20-63)$ minutes $(n=13)$.

(range $0.89-0.99, \mathrm{p}<0.001$ ), and for the group as a whole there was a highly significant relationship between ACSA and volume of feed $(r=0.78, \mathrm{p}<0.001)$.

The magnitude of change in ACSA after complete administration of the feed varied between individuals. The median change was $3 \cdot 1 \mathrm{~cm}^{2}$ representing a $21-$ fold increase, with a range of $1 \cdot 0-10 \cdot 6 \mathrm{~cm}^{2}$. The maximum change in ACSA seen after the feed was not significantly related to the size of the baby.

(ii) Reproducibility of the measurement of ACSA The variation in 10 measurements over one minute in 10 infants was small. The mean (SE) coefficient of variation was $7 \cdot 7 \%(1 \cdot 1)$. This may be compared with a mean increase during gastric filling of $1960 \%$. The maximum deviation observed on any occasion was $-25 \%$ from the mean. Any single result has a $95 \%$ chance of lying within $+/-15 \%$ of the mean of 10 results, and a $90 \%$ chance of lying within $+/-13 \%$.

(iii) Pattern of gastric antral clearance during gastric emptying

A characteristic pattern of gastric antral clearance was seen in 13 of the subjects. In 11 there had been a fall in ACSA when the first postprandial measurement was taken within eight minutes of the end of the feed. In two there appeared to be a lag phase, the first fall in ACSA was seen at 14 and 16 minutes. In all 13 subjects gastric antral clearance was seen as a curvilinear fall in ACSA (fig 3). The shape of the curve was neither linear nor exponential. The median $50 \%$ delta ACSA was 28 minutes (range 20-63). The 50\% delta ACSA was not related to gestation, birth weight, age, or change in ACSA during gastric filling. Our study was not designed to compare differences in $50 \%$ delta ACSA in relation to type of feed. The mean $50 \%$ delta ACSA of the 12 infants receiving breast milk

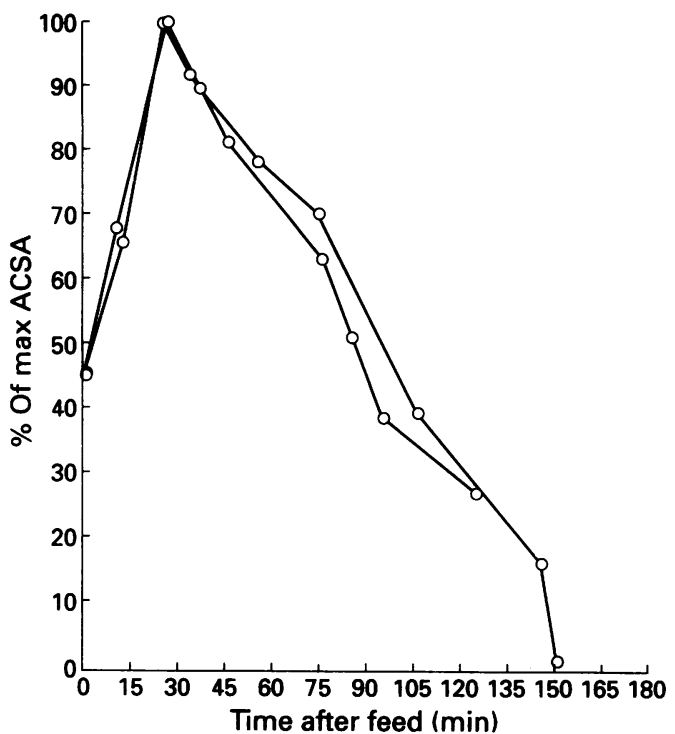

Figure 4 Gastric antral transit: slow rates seen in two subjects. Half gastric antral transit times 92 and 95 minutes.

(35 $\mathrm{min}$ ) was lower than for the five receiving formula ( $45 \cdot 3$ minutes).

The $50 \%$ delta ACSA was significantly correlated with the volume of the feed $(r=0.672$, $\mathrm{p}<0.01$ ) and the infants weight on the day of the study $(r=0.737, \mathrm{p}<0.01)$. These variables were however inter-related, and none of the above was shown to predict significantly $50 \%$ delta ACSA when multiple regression analysis was performed. There was no difference in $50 \%$ delta ACSA between groups who were given their feeds over one minute or more slowly. In 12 subjects the ACSA could be observed to fall by over $90 \%$ of the maximal increase seen after gastric filling. In one subject gas bubbles appeared after the child had been crying so that the last measurement obtained showed a $76 \%$ fall from the maximum change in ACSA at 75 minutes.

In two subjects (fig 4) the ACSA had only risen by 44 and $45 \%$ of its maximum change when measured immediately after the completion of the feed. Maximum ACSA was seen at 25 and 24 minutes and the fall in ACSA was not seen until 32 and 35 minutes, respectively. Both infants had prolonged half gastric antral clearance times of 92 and 95 minutes. In one of these infants the ACSA did not return to prefeed size, and at three hours $11 \mathrm{ml}$ was aspirated from the stomach before the next feed. This was the only infant with a residual volume of more than $2 \mathrm{ml}$.

\section{Discussion}

Gastric emptying in the preterm infant has been the subject of considerable attention but its study has been hampered by the absence of a suitable technique. In this study we present our method of determining gastric antral clearance using serial measurements of the ACSA and evidence of its evaluation and validation. The technique does not directly measure gastric emptying by serial assessment of intragastric volume. Instead it measures the clearance of a feed from the gastric antrum as a 
proxy for gastric emptying. The technique works well in the low birthweight infant. Measurements of ACSA are reproducible, correlate well with gastric volume, and fall during gastric emptying in a manner consistent with recognised patterns of gastric emptying.

There are a number of alternative techniques available for the measurement of gastric emptying. ${ }^{8}$ Isotope scintigraphy, widely seen as the gold standard is not applicable in the neonatal intensive care unit. Other radiographic techniques suffer from similar disadvantages. Measurement of residual volume relies upon complete emptying of the stomach by intragastric tube, a process that may interfere with gastric emptying. Double dye dilution techniques provide an accurate assessment of intragastric volume. ${ }^{9}$ The volumes of dye, although inconsequential in the larger infant, may be an appreciable proportion of the total feed volume in the smaller baby. There is therefore a clear need for a non-invasive test capable of measuring gastric emptying of small volume milk feeds in the infant on the neonatal intensive care unit. ${ }^{1}$

Ultrasound is widely used and readily available in neonatal units. We have therefore developed and evaluated an ultrasonic method of assessing gastric emptying. Serial measurement of a single cross section of the gastric antrum has been validated against barium studies $^{5}$ and scintigraphy ${ }^{8}$ in adults, and in children shows a pattern consistent with gastric emptying. ${ }^{6}$ The only published study in infants gave a pattern which was atypical and difficult to interpret. ${ }^{7}$ Previous experience with ultrasound in adults has shown that complex techniques involving volume reconstruction from multiple images are difficult to perform, ${ }^{34}$ and give similar results to the simpler techniques. ${ }^{5}$ In the preterm infant we chose a single longitudinal section of the antrum fixed by easily definable anatomical landmarks. This is of crucial importance, as the antropyloric area is approximately conical in transverse section, and failure to adhere to anatomical landmarks will invalidate serial measurements. Our technique is relatively simple and provided effective measurements in 15 out of 17 patients without evidence of adverse effects. The technique requires someone practised in abdominal ultrasound, but the necessary equipment is commonly available on neonatal units and we were able to measure gastric antral clearance with feed volumes as little as $8 \mathrm{ml} / \mathrm{kg}$.

Principal among the problems of evaluation of this technique in this population is its validation. As there is no accepted method of measuring gastric emptying to allow comparison, we examined the relationship between ACSA and intragastric volumes, the reliability of the measurement, and the pattern of change in ACSA during gastric emptying. There was a clear linear relationship between intragastric volume and increasing ACSA. This observation is consistent with the hypothesis that passive distension of the gastric antrum, with the patient in the right lateral position, takes place as the stomach fills with milk. Rapid antral distension has been seen by others using a liquid feed in children ${ }^{6}$ and adults, ${ }^{8}$ unlike the delayed antral filling seen with meals containing solids. 510

In order to assess reproducibility serial measurements were made. Ninety five per cent confidence intervals of plus or minus $15 \%$ were seen. Against a change of $2000 \%$ seen during gastric filling this small amount of error is unlikely to affect the measurement of gastric antral clearance.

The pattern of gastric antral clearance had characteristic features. The fall in ACSA occurred within a few minutes of the feed. There was then a curvilinear fall in ACSA that fits with recognised patterns of gastric emptying in the preterm infant. Gastric emptying is not exponential in this group but has a rapid initial phase that, it has been suggested, precedes the onset of mechanisms that regulate emptying, ${ }^{11}$ followed by a slower phase. ${ }^{12-14}$ Our observation of gastric antral clearance therefore its well with anticipated patterns of gastric emptying.

The half gastric antral clearance time may be easily calculated. The median in our population was 28 minutes with a range of 20-63 minutes. This is similar, although a little faster than previous estimates of half gastric emptying times in more mature infants, using dye dilution techniques which lie between 25 and 52 minutes. ${ }^{15-17}$ Early studies suggested that position may be an important determinant of gastric emptying, ${ }^{18}$ but more recent data suggest no difference between prone, supine, right and left lateral in infants between 35 weeks' gestation and term. ${ }^{13}$ One study has shown a difference between breast milk and formula that our study was not designed to demonstrate. ${ }^{11}$

In one infant the ultrasound technique demonstrated incomplete gastric antral clearance, this was the only infant with a residual volume $>2 \mathrm{ml}$. At this point the infant had mild abdominal distension that was relieved by defaecation after a glycerine suppository. The feed volume of $50 \mathrm{ml}$ together with the $10 \mathrm{ml}$ residual was replaced and the study repeated, showing a half gastric antral clearance time of 27 minutes.

In conclusion, we have demonstrated that serial measurement of ACSA provides a relatively simple non-invasive index of gastric emptying in the preterm infant, which we have evaluated and validated in this study. Ultrasonic measurement of gastric antral clearance may be a valuable tool in providing the opportunity to study gastric emptying in the preterm infant, the factors that modulate it, and the investigation of infants in whom gastric emptying is thought to be abnormal.

We are indebted to the nursing staff of the regional neonatal intensive care unit at the Birmingham Maternity Hospital and intensive care unit at the Birmingham Maternity Hospital and
to Mr P H Williams of the medical physics department for his to Mr P H Wical support.

1 Lebenthal E, Siegal M. Understanding gastric emptying implications for feeding the healthy and compromised infant. F Pediatr Gastroenterol Nutr 1985; 4: 1-3.

2 Smith HL, Newell SJ, Puntis JWL, Hollins GW, Booth IW. Use of epigastric impedance recording to measure gastric emptying in two infants with dumping syndrome. European fournal of Gastroenterology and Hepatology 1989;
1: 125-8. 
3 Bateman DN, Whittingham TA. Measurement of gastric emptying by real-time ultrasound. Gut 1982; 23: 524-7. 4 Holt S, Cervantes J, Wilkinson AA, Wallace JHK Measurement of gastric emptying rate in humans by real time ultrasound. Gastroenterology 1986; 90: 918-23.

5 Bolondi L, Bortolotti M, Santi V, Calletti T, Gaiani S, Labo G. Measurement of gastric emptying time by real time ultrasound. Gastroenterology 1985; 89: 752-9.

6 Lambrecht L, Robberecht E, Deschynkel K, Afschrift M. Ultrasonic evaluation of gastric clearing in young infants. Pediatr Radiol 1988; 18: 314-8.

7 LiVoti G, Tulone R, Bruno R, et al . Ultrasonography and gastric emptying: evaluation in infants with gastroesophageal reflux. F Pediatr Gastroenterol Nutr 1992; 14: 397-9.

8 Marzio L, Giacobbe A, Conoscitore P, Facciorusso D, Frusciante V, Modoni S. Evaluation of the use of ultrasonography in the study of liquid gastric emptying. $A m \mathcal{F}$ Gastroenterol 1989; 84: 496-500.

9 George JD. New clinical method of measuring the rate of gastric emptying: the double sampling test meal. Gut 1968; 9: 237-42.

10 Minami H, McCallum RW. The physiology and pathophysiology of gastric emptying in humans. Gastroenterology 1984; 86: $1592-610$.
11 Hunt JN, Smith JL, Jiang CI. Effect of meal volume and energy density on gastric emptying of carbohydrates. Gastroenterology 1985; 89: 1326-30.

12 Cavell B. Gastric emptying in the preterm infant. Acta Paediatr Scand 1979; 68: 725-30.

13 Siegal M, Krantz B, Lebenthal E. Effect of fat and carbohydrate composition on the gastric emptying of isocaloric feeds in premature infants. Gastroenterology 1985; 89: $785-90$

14 Blumenthal I, Ebel A, Pildes RS. Effect of posture on the pattern of stomach emptying in the newborn. Pediatrics 1979; 63: 532-6.

15 Cavell B. Reservoir and emptying function of the stomach of the premature infant. Acta Pediatr Scand Suppl 1982; 296: $60-1$.

16 Pildes RS, Blumenthal I, Ebel A. Stomach emptying in the newborn. Pediatrics 1980; 66: 482-3.

17 Sidebottom R, Curran JS, Williams PR, Kanarek KS, Bramson RT. Effects of long chain vs medium chain triglycerides on gastric emptying in premature infants. f Pediatr 1983; 102: 448-50.

$18 \mathrm{Yu}$ VYH. Effect of body position on gastric emptying in the neonate. Arch Dis Child 1975; 50: 500-5. 